\title{
HELICAL FOFO SNAKE FOR 6D IONIZATION COOLING OF MUONS*
}

\author{
Y. Alexahin, FNAL, Batavia, IL
}

\section{Abstract}

A channel for 6D ionization cooling of muons is described which consists of periodically inclined solenoids of alternating polarity, liquid hydrogen absorbers placed inside solenoids and RF cavities between them. Important feature of such channel (called Helical FOFO snake) is that it can cool simultaneously muons of both signs. Theoretical considerations as well as results of simulations with G4Beamline are presented which show that $200 \mathrm{MHz}$ HFOFO snake has sufficient acceptance to be used for initial $6 \mathrm{D}$ cooling in muon colliders and neutrino factories.

\section{INTRODUCTION}

Ionization cooling is the only method fast enough for cooling of muons which is essential for realization of muon collider, muon beam-based neutrino factories and other experiments involving muons. Unfortunately the longitudinal motion is naturally anti-damped in the most suitable momentum range $(2-300 \mathrm{MeV} / \mathrm{c})$ since the ionization losses decrease with momentum. Though neither neutrino factory nor muon collider requires low longitudinal emittance, longitudinal cooling would be beneficial for both machines due to better transmission and possibility to use higher RF frequency.

Two basic schemes were proposed in order to achieve longitudinal cooling by forcing muons with higher momentum to make a longer path in the absorber so that they lose more energy. This can be realized by creating dispersion in particle positions (without significant path lengthening) and using wedge absorbers (so called RFOFO channel [1]) or by creating sufficiently large path lengthening with momentum and using a homogeneous absorber (Helical Cooling Channel [2]).

In both these schemes the dispersion is generated due to curvature of the reference orbit making these schemes selective w.r.t. the sign of muon charge. A different approach was proposed in [3] where dispersion is produced in the result of periodic perturbation of a straight channel comprised of block solenoids with alternating polarity and RF cavities between them.

This scheme - called "FOFO snake" - is especially attractive due to its technological simplicity and ability to cool both $\mu^{+}$and $\mu^{-}$simultaneously.

In the initial proposal [3] the solenoids were inclined (or displaced) in one plane with a period of 4 solenoids. However, the subsequent analysis showed that such "planar snake" had insufficient transverse dynamic acceptance. It was possible to increase it by making the snake "helical" [4], i.e. by making the transverse magnetic

\footnotetext{
* Work supported by Fermi Research Alliance LLC. Under DEAC02-07CH11359 with the U.S. DOE.
}

alternating solenoids $\quad$ absorbers $\quad$ RF cavities

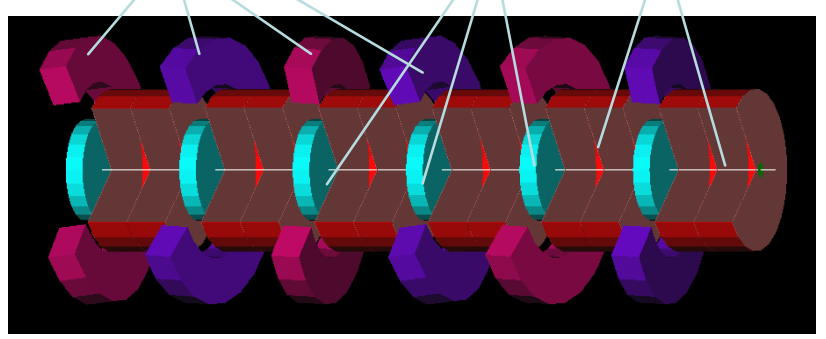

$B\lceil\mathrm{~T}\rceil$

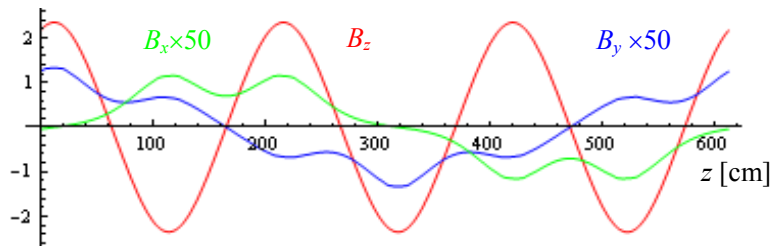

Figure 1. Schematic view and magnetic field distribution along the channel

field direction rotate as seen while moving along the axis (see Fig. 1). The principal difference from the HCC [2] is that the longitudinal field has alternating polarity.

In both planar and helical snakes the orbit displacement from the axis creates problem with equalizing the damping rates of the transverse modes as a result of the "feeddown" effect from the magnetic field nonlinearities. With increase in the solenoid pitch angle one of the transverse modes gets more damping from the other transverse mode than goes to the longitudinal mode limiting both longitudinal and transverse cooling rates which can be achieved in such channels.

In the present report we present a solution to the problem of the transverse damping rates equalization which does not lead to reduction in beam transmission or increase in the equilibrium emittances.

\section{HFOFO SNAKE PRINCIPLES}

The FOFO snake is based on two principles: alternating solenoid focusing and resonant dispersion generation [3].

In a homogeneous longitudinal magnetic field the two transverse modes are Larmor gyration and constant offset. The spread in these offsets (produced e.g. by multiple scattering) can not be reduced by ionization losses since there is no transverse momenta associated with it until the beam exit from the solenoid where the spread in position is converted into a spread in mechanical angular momentum. A well-known solution to this problem is to use solenoids of alternating polarity so that gyration and constant offset interchange periodically assuring equal cooling rates of both transverse modes. 

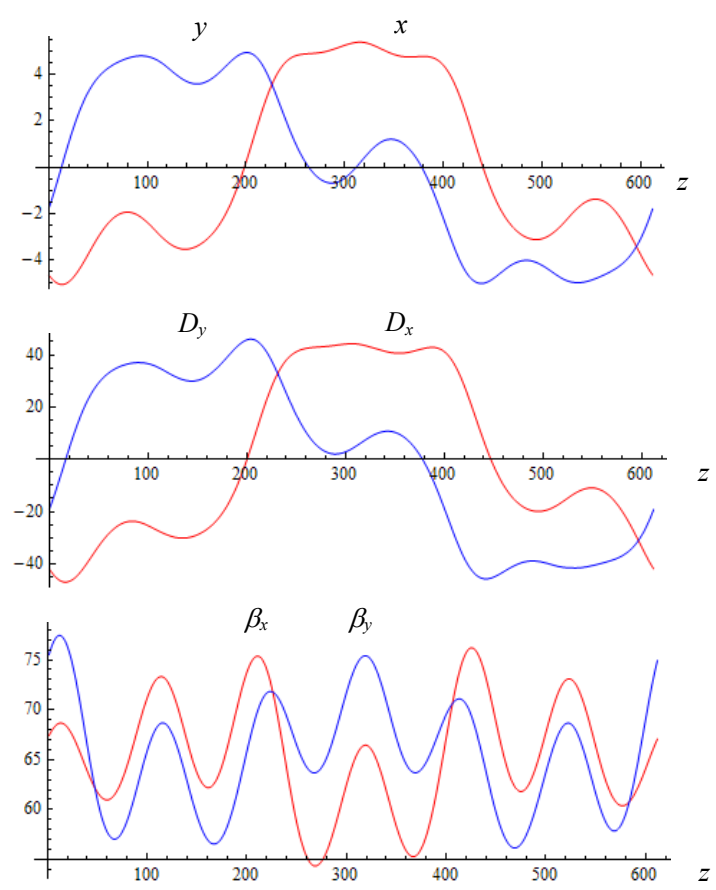

Figure 2. Periodic orbit for $\mu^{+}$(top), dispersion (middle) and $\beta$-functions (bottom), all values in $\mathrm{cm}$.

The second principle - resonant dispersion generation - is easier to explain for a 1D case. Consider a straight focusing channel with periodic driving transverse force. The forced solution (periodic orbit) resonantly depends on the tune per perturbation period: $x_{\text {p.o. }} \sim 1 / \sin \pi Q_{x}$. If there is chromaticity, $Q_{x}{ }^{\prime}=d Q_{x} / d \delta_{p} \neq 0$, we obtain resonant dispersion:

$$
D_{x}=\frac{d x_{\text {p.o. }}}{d \delta_{p}} \approx-\pi Q_{x}^{\prime} x_{\text {p.o. }} \cot \left(\pi Q_{x}\right)
$$

Since naturally the chromaticity is negative, $Q_{x}{ }^{\prime}<0$, we can obtain positive momentum compaction factor $\left(D_{x} \cdot x_{\text {p.o. }}>0\right)$ if the tune is above an integer.

One may expect the momentum acceptance of such a channel to be small, however, as we will see later, it may be sufficiently large due to a higher order chromaticity.

In the case of alternating solenoids the driving transverse field can be obtained by periodically inclining or displacing the solenoids. To make the channel "helical" the solenoids are inclined in rotating planes $x \cdot \cos \left(\phi_{k}\right)+y \cdot \sin \left(\phi_{k}\right)=0, \phi_{k}=\pi\left(1-2 / N_{s}\right)(k-1), k=1,2, \ldots, N_{s}$.

In this report we study particular case of $N_{s}=6$ solenoids per period. Then the transverse tune above integer, $Q_{\perp}>1$, can be obtained with a rather weak focusing which is beneficial for particle stability. The roll angles $\phi_{k}$ in this case are: $0,2 \pi / 3,4 \pi / 3,0,2 \pi / 3,4 \pi / 3$.

\section{ORBIT \& LINEAR OPTICS}

Schematic view of the channel for initial 6D cooling and magnetic field distribution are shown in Fig.1. After some optimization the channel parameters were chosen as follows:
- solenoids: $L=24 \mathrm{~cm}, R_{\text {in }}=60 \mathrm{~cm}, R_{\text {out }}=92 \mathrm{~cm}$,

- RF cavities: $f_{R F}=200 \mathrm{MHz}, L=2 \times 36 \mathrm{~cm}, E_{\max }=16 \mathrm{MV} / \mathrm{m}$,

- absorbers: planar, $L=15 \mathrm{~cm}$, liquid $\mathrm{H}_{2}$.

A $3 \mathrm{~cm}$ technological gap was left between solenoids and cavities so that the one cell length is $102 \mathrm{~cm}$ and $L_{\text {period }}=612 \mathrm{~cm}$. RF cavities are timed at an imaginary particle moving along the axis with constant velocity $v_{0}$.

The properties of the channel were studied using approach described in [5]. Fig. 2 (top) shows periodic trajectory for positive muons in the case of solenoid pitch angle $\vartheta=7 \mathrm{mrad}$, reference momentum $p_{0}=200 \mathrm{MeV} / \mathrm{c}$ and magnetic field amplitude $B_{z \max }=2.35 \mathrm{~T}$. This set of parameters will be used as the baseline set.

Periodic trajectory for $\mu^{-}$looks precisely the same with a shift by half period.

The shape of the dispersion function (Fig.2 middle) almost exactly follows that of the periodic trajectory ensuring that higher energy muons make a longer path (momentum compaction factor $\alpha_{c}>0$ ). Beta-functions (Fig. 2 bottom) have minima in RF cavities and maxima inside absorbers, but the variation is not large.

\begin{tabular}{|c|c|c|c|}
\hline mode & I & II & III \\
\hline tune $Q$ & $1.239+0.012 \mathrm{i}$ & $1.279+0.007 \mathrm{i}$ & $0.181+0.002 \mathrm{i}$ \\
\hline$\varepsilon_{N}, \mathrm{~mm}$ & 3.2 & 4.5 & 6.9 \\
\hline
\end{tabular}

Table 1. Normal mode tunes and emittances

The normal mode tunes and analytically calculated normalized equilibrium emittances for the cited set of parameters are given in Table 1. The tunes were computed from eigenvalues of a one-period transfer matrix, their imaginary parts describe oscillation damping due to regular part of ionization losses. Without stochastic effects the emittances would have been damped as

$$
\frac{d}{d z} \ln \varepsilon_{j}=2 \times \frac{2 \pi}{L_{\text {period }}} \operatorname{Im} Q_{j}
$$

with damping lengths in the considered case being $41 \mathrm{~m}$, $72 \mathrm{~m}$ and $196 \mathrm{~m}$ for the three modes respectively.

Even with plane-parallel absorber surfaces there is an appreciable longitudinal damping due to large dispersion slope, $\mathbf{D}^{\prime}$, which makes higher momentum muons cross the absorber at a larger angle and cover a longer path in it.

Still, there is a significant imbalance in damping rates of the transverse modes.

In Ref.[4] the damping rates ratios of all three modes were studied vs. solenoid inclination angle $\vartheta$, (conical) wedge angle of the absorbers and the transverse tune (controlled by magnetic field strength for a given momentum and channel geometry). It was shown that equalization of the damping rates is in principle possible at sufficiently large solenoid inclination and absorber wedge angles. Large inclination angle, however, may reduce the momentum acceptance.

\section{MOMENTUM ACCEPTANCE}

To get an insight into dynamics at large momentum deviations we can assume the longitudinal motion to be 
slow and calculate betatron tunes and relative orbit length $\lambda=s(p) / L_{\text {period }}$ for a constant momentum $p$ (Fig.3).
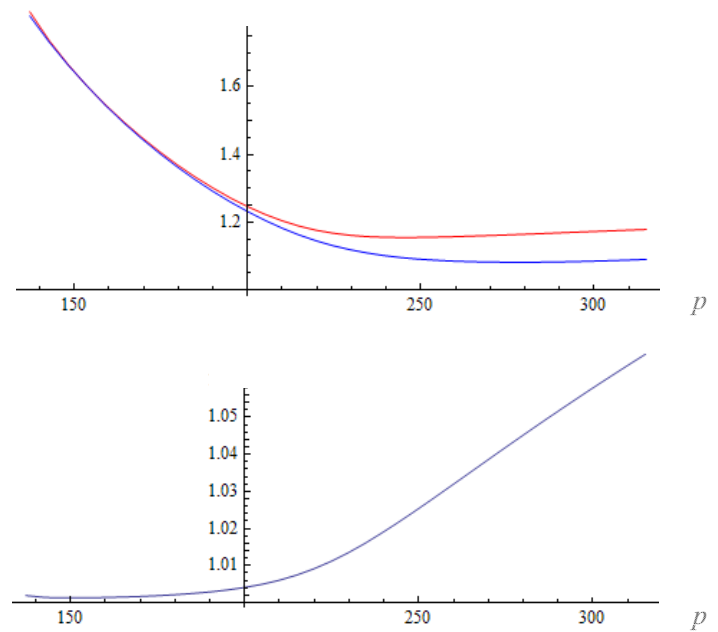

Figure 3. Betatron tunes (top) and relative orbit length (bottom) vs muon momentum ( $\mathrm{MeV} / \mathrm{c}$ ) for the solenoid pitch angle $\theta=7 \mathrm{mrad}$.

First of all, Fig. 3 reveals a surprising phenomenon of the transverse tunes "repulsion" from the integer resonance making the energy width of the betatron passband very large [4]. The orbit length (and its maximum offset) increases just linearly with momentum.

To find the momentum width of the longitudinal separatrix let us introduce canonical variables

$$
\delta_{p}=\left(\gamma-\gamma_{0}\right) / \beta_{0}^{2} \gamma_{0}, \quad u=z-c \beta_{0} t
$$

Kinetic part of the longitudinal Hamiltonian in the considered quasi-stationary approximation is*

$$
K\left(\delta_{p}\right)=\int^{\delta_{p}}\left[1-\lambda(\xi) \frac{\beta_{0}}{\beta(\xi)}\right] d \xi
$$

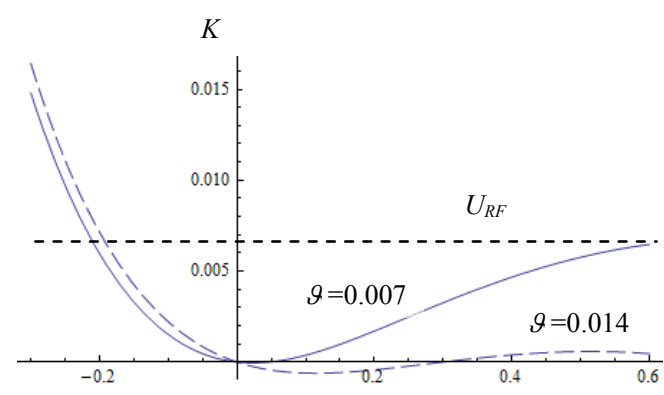

$\delta_{p}$

Figure 4. Effective longitudinal kinetic energy.

Fig.4 shows large asymmetry in the effective kinetic energy $K$. Due to this asymmetry the separatrix area initially increases with the inclination angle $\vartheta$ until the maximum value of $K$ is lowered to the RF bucket height, $U_{R F}$. At larger values of $\vartheta$ the momentum acceptance start

*) It can be easily verified that for small $\delta_{p}$ and $\lambda(0)=1$ eq.(3) yields standard expression for the slippage factor to decrease. The optimum value for the chosen parameters of the channel is close to $\vartheta=7 \mathrm{mrad}$.

\section{DAMPING RATE EQUALIZATION}

With further increase in the solenoid inclination angle $\vartheta$ being ruled out as a means for making damping rates equal, R.Palmer proposed to add constant component to solenoidal field so that the transverse modes were better mixed [6]. This idea worked remarkably well from the point of view of damping rate equalization but exacerbated the problem with transmission and increased beam emittances due to strong beta-beat [5].

It turned out that the problem can be solved by adding a quadrupole field, constant or modulated along the channel so that the maxima were reached inside solenoids. A gradient of just $0.057 \mathrm{~T} / \mathrm{m}$ is sufficient to equalize the transverse modes damping rates (see Table 2).

\begin{tabular}{|c|c|c|c|}
\hline mode & I & II & III \\
\hline tune $Q$ & $1.240+0.009 \mathrm{i}$ & $1.286+0.009 \mathrm{i}$ & $0.178+0.004 \mathrm{i}$ \\
\hline$\varepsilon_{N}, \mathrm{~mm}$ & 3.9 & 3.8 & 4.7 \\
\hline
\end{tabular}

Table 2. Normal mode tunes and emittances in the presence of constant quadrupole field.

Comparison with Table 1 shows that the quadrupole field not only equalizes the transverse modes damping rates and emittances, but also reduces the equilibrium longitudinal emittance by one third while keeping the sum of the transverse emittances constant. This can be explained by increase in the dispersion function and at the same time reduction in the average values of betafunctions at absorbers (see Fig.5).
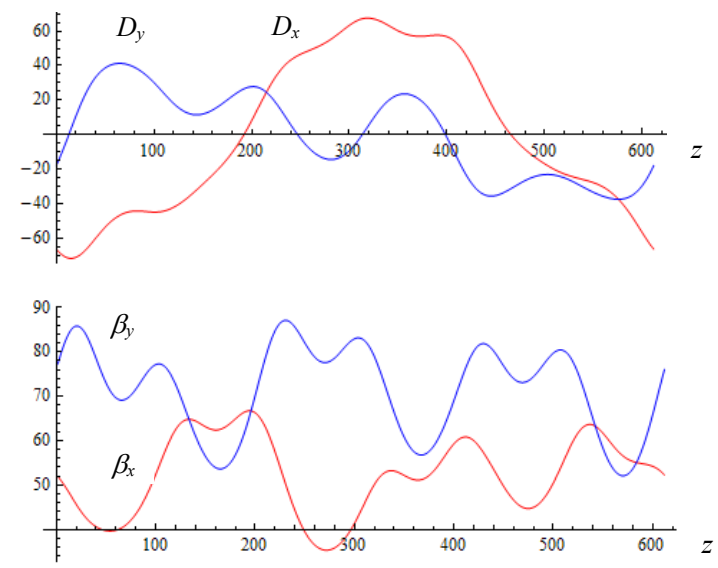

Figure 5. Dispersion (top) and $\beta$-functions (bottom) in the presence of constant quadrupole field.

Interestingly enough the effect of the quadrupole field does not depend on its roll angle (and therefore its polarity) so that it works equally well for both $\mu^{+}$and $\mu^{-}$.

\section{TRACKING SIMULATIONS}

Nonlinear motion in the channel was first studied analytically and numerically using Mathematica-based code MICCD specially developed for this purpose [5]. 
The most prominent nonlinear effect found analytically is correlation between average particle momentum and amplitude of oscillations. For the baseline parameters

$$
\delta_{p}=\delta_{p 0}+0.042 J_{1}+0.013 J_{2}+0.039 J_{3}
$$

where $\delta_{p 0}$ is the periodic orbit value and $J_{m}$ is the action variable for the $m$-th normal mode.

This correlation has simple explanation: particles with larger oscillation amplitude make longer path and must have higher velocity to keep in phase with RF. In a real channel such correlation will develop naturally during capture in RF buckets, but for tracking simulations it must be introduced into the initial conditions to avoid excitation of large amplitude longitudinal oscillations which will cause loss of particles with large initial transverse amplitudes.

To prepare the ensemble for tracking simulations the generating functions were calculated of the Lie-transform from the space of "true" nonlinear invariants $J_{m}$ to the physical phase space. 1771 particles were evenly distributed in tetrahedron

$$
\left(J_{1}+J_{2}\right) / 2.6+J_{3} / 4<1(\mathrm{~cm})
$$

with random phases. Tracking without decays and stochastic processes showed fast cooling with transmission over 25 periods (153m) about $97 \%$ [4].
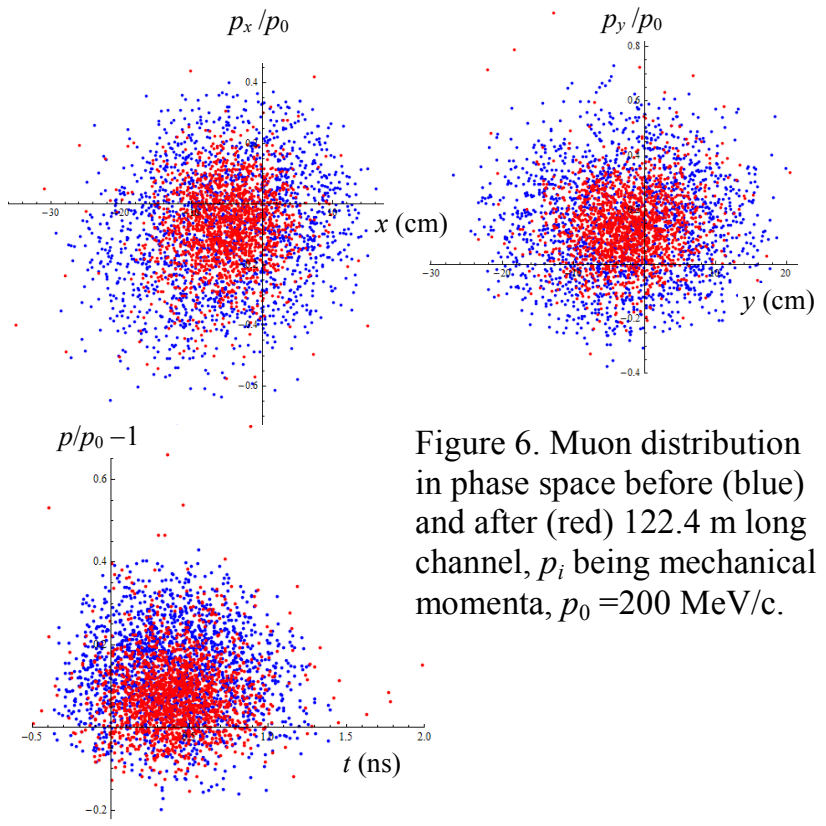

Figure 6. Muon distribution in phase space before (blue) and after (red) $122.4 \mathrm{~m}$ long channel, $p_{i}$ being mechanical momenta, $p_{0}=200 \mathrm{MeV} / \mathrm{c}$.

To study the effect of stochastic processes the latest version 1.16 of G4beamline [7] was used. Fig. 6 shows initial and final particle distribution after 20 periods $(122.4 \mathrm{~m})$ in absence of the quadrupole field.

The transmission over this distance calculated with stochastic processes (G4BL) and without them (G4BL and MICCD) is shown in Fig. 7. One may note a good agreement between the two codes. Decay losses were not accounted for in this plot. They reach up to $9 \%$ over 20 periods, so that the total losses are about $14 \%$.

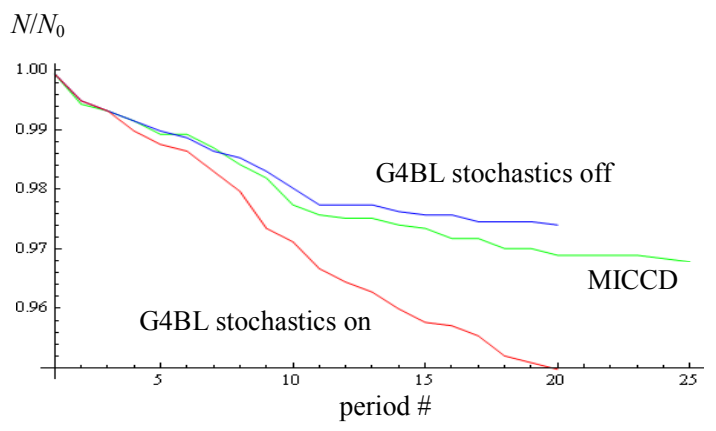

Figure 7. Transmission in absence of decay losses computed with MICCD and G4BL for channel without auadrupole field.

To obtain emittance evolution from the tracking data the following recipe was applied:

- using the transfer matrix eigenvectors compute the normal mode amplitudes $a_{m}$ for each particle after each lattice period,

- subtract average values over the ensemble of particles (representing error in the periodic orbit position),

- calculate distribution in linear action variables $I_{m}=\left|a_{m}\right|^{2}$,

- fit the distribution with exponential function

$$
f \sim \exp \left(-\sum \frac{I_{m}}{\varepsilon_{m}}\right)
$$

- find $\varepsilon_{m N}=\beta_{0} \gamma_{0} \varepsilon_{m}, m=1,2,3$.

The emittance evolution obtained in this way with and without quadrupole field is presented in Fig. 8. The fact that there is no initial jump in emittances confirms the validity of the initial ensemble preparation with the help of the Lie-transform perturbation theory. One can also see that the damping rate equalization with a quad renders transverse mode emittances equal.

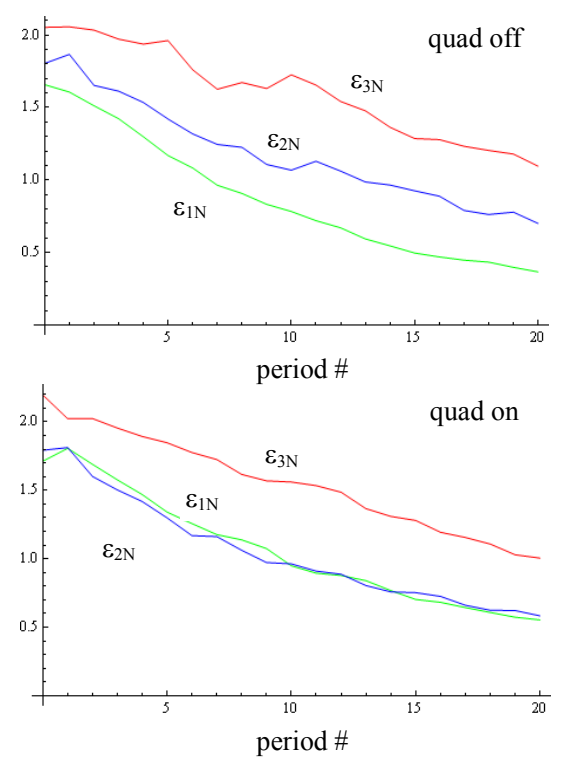

Figure 8. Normalized emittances $(\mathrm{cm})$ vs. period number as computed with G4BL with quadrupole field off (top) and on (bottom). 


\begin{tabular}{|l|l|l|l|l|l|}
\hline \multicolumn{2}{|c|}{} & $\varepsilon_{1 \mathrm{~N}}, \mathrm{~cm}$ & $\varepsilon_{2 \mathrm{~N}}, \mathrm{~cm}$ & $\varepsilon_{3 \mathrm{~N}}, \mathrm{~cm}$ & $\varepsilon_{6 \mathrm{D}}, \mathrm{cm}^{3}$ \\
\hline \multirow{2}{*}{$\begin{array}{l}\text { quad } \\
\text { off }\end{array}$} & initial & 1.658 & 1.804 & 2.054 & 6.144 \\
\cline { 2 - 6 } & final & 0.367 & 0.701 & 1.096 & 0.282 \\
\hline \multirow{2}{*}{$\begin{array}{l}\text { ouad } \\
\text { on }\end{array}$} & initial & 1.713 & 1.793 & 2.197 & 6.749 \\
\cline { 2 - 6 } & final & 0.554 & 0.584 & 1.006 & 0.326 \\
\hline
\end{tabular}

Table 3. Initial and final normalized emittances.

Initial and final values of emittances of the three normal modes are given in Table 3. It is interesting that the initial values appear different depending on whether the quadrupole field is on or off though the underlying distribution in nonlinear invariants (6) was the same. This can be explained by change in the Lie-transform generating functions that determine distribution (6) projection onto the physical phase space. However, the ratio of the final to initial values of $6 \mathrm{D}$ emittance is about the same in both cases, as well as the transmission.

From these data one can calculate the 6D cooling efficiency as introduced by R.Palmer:

$$
Q_{6 \mathrm{D}}=\ln \frac{\varepsilon_{6 \mathrm{D}}(L)}{\varepsilon_{6 \mathrm{D}}(0)} / \ln \frac{N(L)}{N(0)},
$$

which turns out to be $Q_{6 \mathrm{D}}=20$ in both cases.

\section{Effect of the walls}

In the above analysis the contribution to ionization losses and scattering from the absorber and RF cavity walls was neglected. Since the walls are made from higher $Z$ materials than the hydrogen, their contribution may significantly spoil the performance.

For the present study the twin RF cavities were assumed to share the inner wall so that there were three $0.38 \mathrm{~mm}$ thick Be walls for a pair of cavities.

The absorber walls can be made from the aluminum alloy 2090-T81 used for the Space Shuttle hydrogen tanks [8]. It has almost twice the strength of the 6061-T6 alloy proposed for the MICE absorbers [9]. The thickness can be made correspondingly smaller, $0.1 \mathrm{~mm}$ vs. $0.18 \mathrm{~mm}$.

In the study the absorber walls were introduced first and then the cavity walls added. To keep the average energy and the transverse dynamics unchanged the RF voltage was increased accordingly. Table 4 cites damping rates and equilibrium emittances computed with MICCD without quadrupole field as well as the required RF amplitude.

\begin{tabular}{|r|c|c|c|}
\hline & $\operatorname{Im} Q_{m} \times 10^{3}$ & $\varepsilon_{m N}, \mathrm{~mm}$ & $E_{\mathrm{RF}}, \mathrm{MV} / \mathrm{m}$ \\
\hline no walls & $11.8 / 6.8 / 2.5$ & $3.2 / 4.5 / 6.9$ & 16.0 \\
\hline Abs. walls & $12.0 / 6.8 / 2.6$ & $3.7 / 5.1 / 7.5$ & 16.3 \\
\hline+ RF walls & $13.0 / 6.8 / 3.3$ & $4.0 / 5.8 / 7.3$ & 17.5 \\
\hline
\end{tabular}

Table 4. Damping rates and equilibrium emittances without and with absorber and cavity walls.

The effect of the walls is quite moderate: $\sim 25 \%$ increase in the transverse emittances and $\sim 6 \%$ increase in the longitudinal emittance. Tracking with G4BL shows even smaller increase, the final values for the three modes after 20 periods being $0.363,0.777$ and $1.022 \mathrm{~cm}$ which should be compared with values in the third row of
Table 3. The first and the third mode emittances actually decreased owing to higher RF gradient. There was no appreciable effect of the walls on transmission.

\section{SUMMARY \& OUTLOOK}

- Helical FOFO snake has the ability of 6D cooling both $\mu^{+}$and $\mu^{-}$simultaneously with high average efficiency $Q_{6 \mathrm{D}}=20$.

- HFOFO with $200 \mathrm{MHz}$ double RF cavities has sufficient acceptance to be used for the initial cooling of muons after buncher/rotator.

- G4BL final emittances after a $122.4 \mathrm{~m}$ long channel are $\varepsilon_{\perp \mathrm{N}}=6 \mathrm{~mm}, \varepsilon_{\| \mathrm{N}}=10 \mathrm{~mm}$ with contribution from the absorber and cavity walls included.

- By scaling down the channel parameters (and correspondingly increasing the RF frequency) it seems possible to achieve emittances $\varepsilon_{\perp \mathrm{N}} \sim 1 \mathrm{~mm}, \varepsilon_{\| \mathrm{N}} \sim 1.5 \mathrm{~mm}$ with $800 \mathrm{MHz}$ single-cell cavities.

- It seems possible to achieve higher cooling rates and smaller transverse emittances by moderately pressurizing RF cavities with $\mathrm{GH}_{2}$ as proposed by M. Zisman [10].

- The work has started on a low-beta HFOFO which would allow to achieve a factor of 5 smaller emittances.

The author is grateful to V. Balbekov, D. Kaplan, R. Palmer and V. Shiltsev for helpful discussions.

\section{REFERENCES}

[1] R. Palmer et al. PRSTAB 8, 061003 (2005).

[2] Y. Derbenev, R. Johnson, PRSTAB 8, 041002 (2005).

[3] Y. Alexahin, R. Palmer, K. Yonehara, PAC07, THPAN106 (2007).

[4] Y. Alexahin, PAC09, TU3PBC04 (2009).

[5] Y. Alexahin, PAC09, TH5PFP055 (2009).

[6] R. Palmer, private communication

[7] T. Roberts, http://g4beamline.muonsinc.com

[8] D. Summers, private communication

[9] D.E.Baynham et al., LBNL-58999 (2005).

[10] M. Zisman, this Workshop 